\title{
Images
}

\section{Saddle pulmonary emboli: an unusual presentation}

\author{
Truptesh H. Kothari, MD, MS; ${ }^{*}$ Shivangi Kothari, MD; ${ }^{\dagger}$ Mahima Pandey, MD; ${ }^{*}$ Harshit Khara, MD; ${ }^{\ddagger}$ \\ Nishant Dhungel, MD*
}

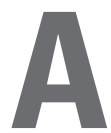

38-year-old man with a history of polyposis syndrome diagnosed 3 years previously, with poor compliance for follow-up, presented to the emergency department with symptoms of retrosternal chest pain associated with dizziness and shortness of breath. His blood pressure was $94 / 43 \mathrm{~mm} \mathrm{Hg}$, his pulse was 123 beats/min and he had an oxygen saturation of $84 \%$ on room air. The patient's initial laboratory results showed a hemoglobin of $80 \mathrm{~g} / \mathrm{L}$ and blood gas with a $\mathrm{pH}$ of 7.23 . He had a normal chest radiograph and electrocardiogram, but had an elevated troponin I at $0.12 \mu \mathrm{g} / \mathrm{L}$. He was given acetylsalicylic acid for suspicion of acute coronary syndrome. On physical examination, the patient was found to have right calf tenderness. With this finding and the presenting symptoms, he underwent computed tomography angiography (CTA) of the chest. The chest CTA showed a massive saddle

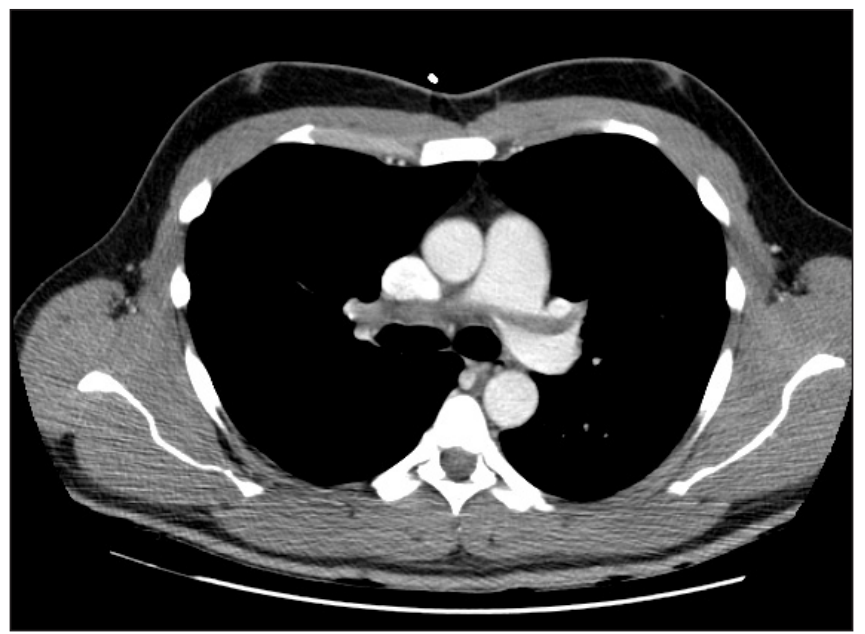

Fig. 1. Computed tomography scan with pulmonary embolism protocol showing entire involvement of pulmonary artery bilaterally, suggestive of saddle emboli. embolus with a filling defect completely occluding the right pulmonary artery and extending through the main pulmonary artery segment to involve the left pulmonary artery. There were also diffuse filling defects involving bilateral pulmonary segmental arteries (Fig. 1 and Fig. 2). The patient received alteplase and underwent a workup for a hypercoagulable state. His workup revealed positive anticardiolipin antibodies and factor $\mathrm{V}$ Leiden. The Doppler ultrasound of his lower extremities showed an extensive thrombus measuring more than $6 \mathrm{~cm}$ extending in the right superficial femoral vein. The patient was then referred for placement of an inferior vena cava filter.

\section{DISCUSSION}

Pulmonary embolism, usually accompanied by deep

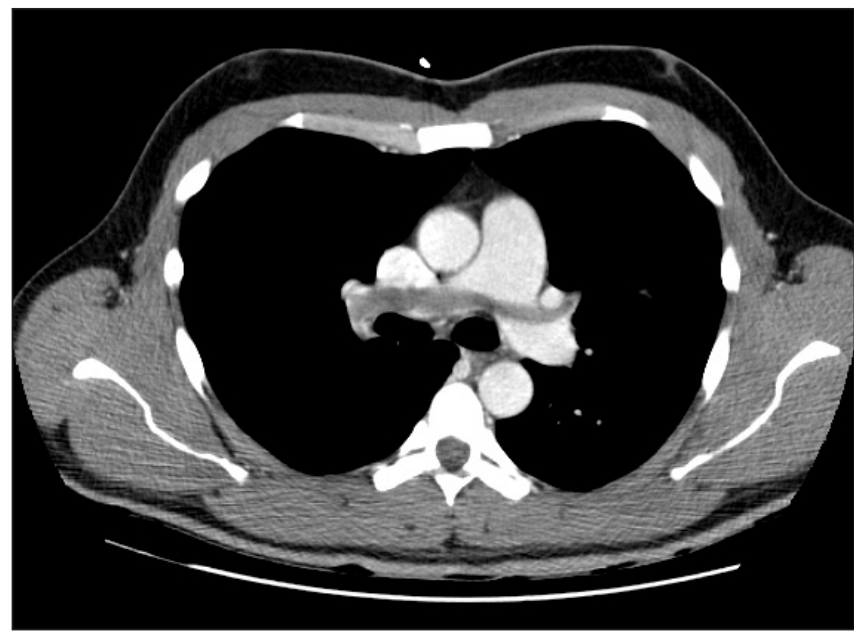

Fig. 2. Computed tomography scan with pulmonary embolism protocol at the thoracic level showing saddle emboli leading to complete obliteration of the pulmonary arteries.

From the *Department of Internal Medicine, J.J. Peters Medical Center and Mount Sinai Program, Bronx, NY, the †Department of Gastroenterology, St. Joseph's Regional Medical Center, Paterson, NJ, and the ‡Department of Internal Medicine, St. Peters Medical Center, New Brunswick, NJ

Submitted Feb. 23, 2009; Accepted Mar. 10, 2009

This article has not been peer reviewed.

CJEM 2009;11(6):558-9 
venous thrombosis, represents the most common manifestation of the antiphospholipid syndrome (APS) and may be the earliest manifestation of the disease. There is no difference clinically from an embolism in patients without APS. Recurrent pulmonary emboli can lead to pulmonary hypertension, which in severe cases can be associated with tricuspid insufficiency. A variety of patients with APS can present with widespread thrombotic occlusion affecting small pulmonary arteries or capillary lumens of the alveoli. However, involvement of major pulmonary artery is a rare manifestation. Venous thromboembolism, mainly of the lower extremities, can occur in up to $55 \%$ of patients with APS.

Competing interests: None declared.

Keywords: pulmonary embolus, saddle embolus, factor V Leiden

Correspondence to: Dr. Truptesh Kothari, J.J. Peters Medical Center and Mount Sinai Program, 130 W. Kingsbridge Rd., Bronx NY 10468; itskots@gmail.com

\section{SERVICE INFORMATION}

\section{Subscription and sales}

The Canadian Journal of Emergency Medicine (CJEM) is supplied to paid-up members of the Canadian Association of Emergency Physicians (CAEP) as a perquisite of membership; others may subscribe yearly. Rates for 2009 (6 issues): Canada (personal), \$225; Canadian institutions, \$399; outside Canada (personal), US\$254; US institutions, US\$449. Contact the CAEP office (800 463-1158). Single copies of current year issues $\$ 50$; back issues $\$ 50$ (subject to availability). Payment should be made to CAEP in funds specified drawn on a Canadian or US bank. VISA and MasterCard are also accepted.

\section{Change of address}

We require 6-8 weeks' notice to ensure uninterrupted service. Please fax your current mailing label, new address and effective date to 613 523-0190 or email: cjem@caep.ca. Send US and foreign address changes to International Media Services (IMS) of New York, 100 Walnut St., \#3, PO Box 1518, Champlain NY 12919-1518.

\section{Reprints}

Bulk reprints of CJEM articles are available in minimum quantities of 50 . For information or orders, please contact the reprint coordinator, Janis Murrey, 800 663-7336 or 613 731-8610 x2110, fax 613 565-7704, janis.murrey@cma.ca.

\section{Electronic availability}

CJEM is available on the CAEP website (cjem-online.ca).

\section{Indexing}

CJEM is indexed by MEDLINE/PubMed, EMBASE,
CINAHL,International Pharmaceutical Abstracts (IPA), BIOME/OMNI, Scirus, Cochrane Prehospital and Emergency Health Field, and PubsHub.com

\section{Copyright and permissions}

Copyright for all material is held by CJEM or its licensors unless otherwise indicated. You may reproduce or otherwise use material from this Journal only in accordance with Canadian copyright law and provided that credit is given to the original source. In the case of photocopying or other reprographic copying, please contact the Canadian Copyright Licensing Agency (Access Copyright): 800 893-5777; accesscopyright.ca. For any other use, including republishing, redistribution, storage in a retrieval system or transmission, in any form or by any means, please contact the CJEM Editorial Office, c/o Penelope Gray-Allan, Managing Editor, Canadian Journal of Emergency Medicine, Department of Emergency Medicine, University of British Columbia, c/o Department of Emergency Medicine, Vancouver General Hospital, 855 West 12th Ave., Vancouver BC V5Z 1M9; cjem@caep.ca

\section{Instructions for authors}

Published in the January issue of each volume. The most recent version is available online at cjem-online.ca.

\section{Advertising}

Classified ads: Contact Bev Kirkpatrick or Deborah Rodd, CJEM, 1867 Alta Vista Dr., Ottawa ON K1G 5W8; 800663 7336 or $613731-8610 \times 2127 / 2314$, fax $613565-7488$, advertising@cma.ca. See Classified Advertising section of the Journal for information on rates. Display ads: Contact Deborah Woodman (see preceding information); x2159. 